\title{
Schumann resonance observation in China and anomalous disturbance possibly associated with Tohoku M9.0 earthquake
}

\author{
Xinyang Ouyang $\cdot$ Xuemin Zhang $\cdot$ A. P. Nickolaenko $\cdot$ \\ M. Hayakawa $\cdot$ Xuhui Shen $\cdot$ Yuanqing Miao
}

Received: 8 March 2013/Accepted: 6 May 2013/Published online: 30 October 2013

(C) The Seismological Society of China, Institute of Geophysics, China Earthquake Administration, and Springer-Verlag Berlin Heidelberg 2013

\begin{abstract}
Schumann resonance (SR) is an electromagnetic resonance phenomenon in the Earth-ionosphere cavity exited by global lightning activities when the wavelength matches the circumference of the Earth, and the lowest four peak frequencies of SR are about 8, 14, 20, and $26 \mathrm{~Hz}$. This article presents the new observational data of SR in China. The observations of two horizontal magnetic components $\left(B_{\mathrm{NS}}\right.$ and $\left.B_{\mathrm{EW}}\right)$ in the frequency band range of $3-29 \mathrm{~Hz}$ at Yongsheng observatory $\left(26.7^{\circ} \mathrm{N}\right.$, $100.8^{\circ} \mathrm{E}$ ) in southwestern China were mainly analyzed. It is found that the SR amplitudes at peak frequencies in $B_{\mathrm{NS}}$ and $B_{\text {EW }}$ components all showed diurnal and seasonal variations, and that the SR amplitude in $B_{\mathrm{NS}}$ component is always higher than that in $B_{\mathrm{EW}}$ component. Diurnal variation of SR amplitude around equinoxes and solstices in
\end{abstract}

X. Ouyang $(\bowtie) \cdot X$. Zhang $\cdot$ X. Shen

Institute of Earthquake Science, China Earthquake

Administration, 63 Fuxing Road, P.O.Box 166, Beijing 100036,

China

e-mail: oyxy@seis.ac.cn

X. Ouyang

School of Earth and Space Sciences, Peking University,

Beijing 100871, China

A. P. Nickolaenko

Usikov Institute of Radiophysics and Electronics, Kharkov, Ukraine

M. Hayakawa

Hayakawa Institute of Seismo Electromagnetics Co. Ltd., UEC Incubation Center, 1-5-1 Chofugaoka Chofu, Tokyo 182-8585, Japan

Y. Miao

DFH Satellite Co., Ltd., China Academy of Space Technology, Beijing 100094, China
$B_{\mathrm{NS}}$ component is related to active intervals of three global thunderstorm centers, while SR amplitude in $B_{\mathrm{EW}}$ component is the most significant at around $16 \mathrm{LT}$, corresponding to Asian center. SR amplitudes both in $B_{\mathrm{NS}}$ and $B_{\mathrm{EW}}$ components increase in the rainy season from May to September. In addition, the SR anomalies in association with the 2011 Japan earthquake are exhibited. The anomalous effect was characterized by an increase in amplitude at the lowest four SR modes beginning at 4 days before this earthquake. Upon analyzing the wave interference between the direct wave and disturbed wave scattered by localized modification of lower ionosphere over the epicenter, Asian and African thunderstorm centers are found to contribute to anomalous effect observed at Yongsheng station. Modeling results of SR regular and disturbed spectra at different local times led to the similar conclusion.

Keywords Schumann resonance amplitude . Thunderstorm center · Anomalous Schumann resonance

\section{Introduction}

Schumann predicted an electromagnetic resonance in the cavity formed by the Earth's surface and its ionosphere (Schumann 1952). Waves of certain frequencies generated by lightning activities travel along the surface of the Earth and become accentuated at about 8, 14, 20, and $26 \mathrm{~Hz}$, which are referred to as Schumann resonance (SR). The SR amplitude and resonant frequencies change because of the vertical extension of the waveguide and the modifying conductivity of lower ionosphere, and they are also related to the distance of observing stations from the sites of three lightning centers which are the main source of SR 
(Nickolaenko and Hayakawa 2002; Schlegel and Füllekrug 2002). Anomalous SR possibly associated with earthquake was first reported by Hayakawa et al. (2005). They found that amplitude of SR at the fourth mode increased, and the fourth peak frequency shifted more than $1 \mathrm{~Hz}$ between $B_{\mathrm{NS}}$ (north-south component of magnetic field) and $B_{\text {EW }}$ (eastwest component) before Chi-chi, Taiwan earthquake. Ohta et al. (2006) carried out a further statistical study on the relation between anomalous SR observed in Japan and earthquakes in Taiwan during 6 years, and found a good correlation between anomalous effects and earthquakes which occurred on the land. Hayakawa et al. (2008) presented another case study of anomalous SR 3 days before an earthquake in Taiwan occurred on December 26, 2006.

As for the mechanism of SR anomaly, some publications suggested that it might be caused by the interference between the direct path from American/African/Asian thunderstorm source to the observatory and the disturbed path scattered by the perturbation in the ionosphere (Hayakawa et al. 2005; Ohta et al. 2006). Nickolaenko et al. (2006) modeled the amplitude of SR changes when there was a localized decrease in the lower ionosphere height over the epicenter, and found that the modeling results were qualitatively similar to the observations.

As SR is mainly used to monitor global lightning activities, its application in earthquake science is a new subject. The reports of anomalous SR associated with earthquakes are still very few until now. This article will present SR observation in China, and show some anomalous effects which may be related to the 2011 Japan earthquake. Finally, we discuss the mechanism of SR anomalies and present modeling results to verify the possible effects on SR from different sources.

\section{Observatories and data acquisition}

We established four observatories in Yunnan province, southwestern China since 2010. They are located at Qiaojia county (QJ: $\left.26.9^{\circ} \mathrm{N}, 102.9^{\circ} \mathrm{E}\right)$, Tonghai county (TH: $24.1^{\circ} \mathrm{N}$, $102.8^{\circ} \mathrm{E}$ ), Yongsheng county (YS: $26.7^{\circ} \mathrm{N}, 100.8^{\circ} \mathrm{E}$ ), and Mangshi (MS: $24.4^{\circ} \mathrm{N}, 98.6^{\circ} \mathrm{E}$ ). The detector consists of three orthogonal search coils with a one-meter-long core, which can measure three components of magnetic field (i.e., $B_{\mathrm{NS}}$ (north-south) which is sensitive to the waves propagating in $\mathrm{EW}$ direction, $B_{\mathrm{EW}}$ (east-west) which is sensitive to the waves in the meridian plane, and $\mathrm{B}_{\mathrm{v}}$ (vertical)) in the frequency range of 3-29 $\mathrm{Hz}$ with a sampling frequency of $100 \mathrm{~Hz}$. Data acquisition at QJ, TH, and YS began from August 2010, and MS began to provide data from December 2011. In this article, we mainly analyze SR variation characteristics in the normal condition and during the Japan earthquake in 2011. MS observatory was built at the end of
2011, and it does not cover the period of 2011 Japan earthquake. We can use data from other three observatories, i.e., QJ, TH, and YS. However, because of some unknown local interference, data from $\mathrm{TH}$ and QJ observatories are mostly spoiled. Figure 1 presents corresponding spectra of one day's record in 2011 from QJ, TH, and YS observatories. We use the same method to obtain spectra for data of all the three stations, and frequency resolution of these spectrum is about $0.1 \mathrm{~Hz}$. One cannot discern various SR modes from the spectra of both $B_{\mathrm{NS}}$ and $B_{\mathrm{EW}}$ components at TH station from Fig. 1c, d. Although there are four clear SR modes in $B_{\mathrm{NS}}$ component at QJ station (see Fig. 1a), it is very difficult to distinguish SR modes from spectrum of $B_{\mathrm{EW}}$ component at QJ station (Fig. 1b). For spectrum recorded at YS station (Fig. 1e, f), it is easier to notice the lowest four SR modes in two components. Hence, we just use the data recorded at YS observatory with good continuity and relatively low noise in this study.

\section{Observational results}

\subsection{Background characteristics of SR amplitude at YS observatory}

Many articles showed that the amplitudes of different SR modes presented diurnal and seasonal variations at different observation sites all over the world (Price and Melnikov 2004; Roldugin et al. 2004; Rossi et al. 2007; Sátori and Zieger 1996; Sekiguchi et al. 2008; Sentman and Fraser 1991). The diurnal variation of SR amplitude may be related to dominant intervals of three global lightning centers and local height of D region (Price and Melnikov 2004; Sentman and Fraser 1991). The seasonal variation of SR may be correlated to the periodical enhancement of thunderstorms in summer every year.

\subsubsection{Diurnal variation of SR amplitude}

Data analysis showed that SR amplitudes at the lowest four peak frequencies both in $B_{\mathrm{NS}}$ and $B_{\mathrm{EW}}$ components are found to have a clear diurnal variation. We used SR data 2 days before and after the equinoxes and solstices in 2011 to obtain a diurnal variation of SR amplitude in four seasons. The first $\mathrm{SR}$ mode is the most stable one. Figure 2 presents the amplitudes of the first $\mathrm{SR}$ mode both in $B_{\mathrm{NS}}$ and $B_{\mathrm{EW}}$ components around spring and autumn equinoxes (Fig. $2 \mathrm{a}$, c), and summer and winter solstices (Fig. 2b, d). The dashdotted lines with circles represent the $B_{\mathrm{NS}}$ component and the dash-dotted curves with asterisks the $B_{\mathrm{EW}}$ component. It can be seen from the figures that in different seasons, the shapes of diurnal variation of SR amplitude at YS observatory change significantly, and there are also different 
(a) 2011/03/01 NS Power spectrum at QJ

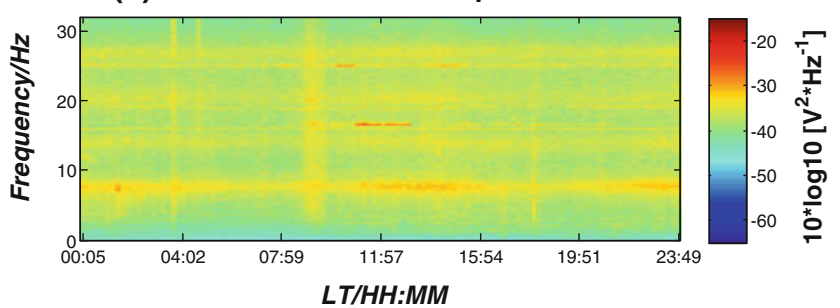

(c) 2011/03/01 NS Power spectrum at TH

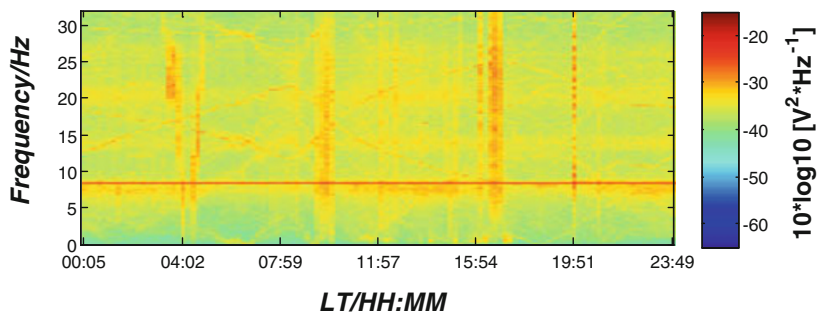

(e) 2011/03/01 NS Power spectrum at YS

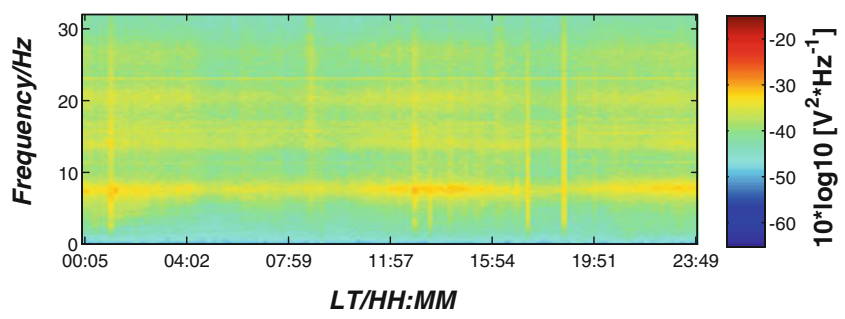

(b) 2011/03/01 EW Power spectrum at QJ

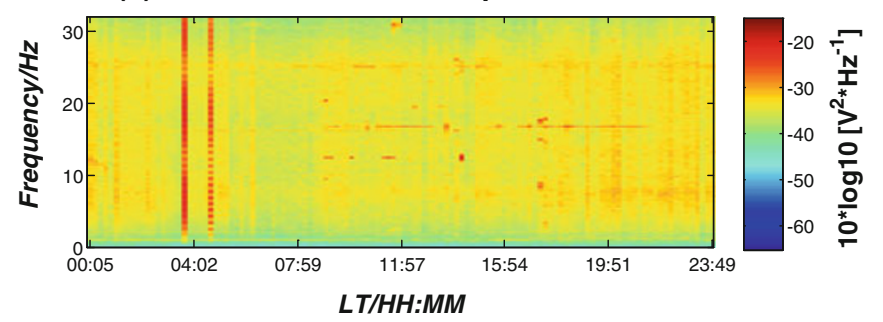

(d) 2011/03/01 EW Power spectrum at TH

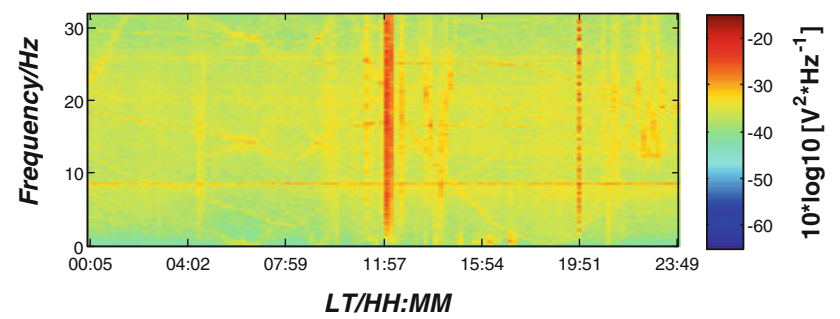

(f) 2011/03/01 EW Power spectrum at YS

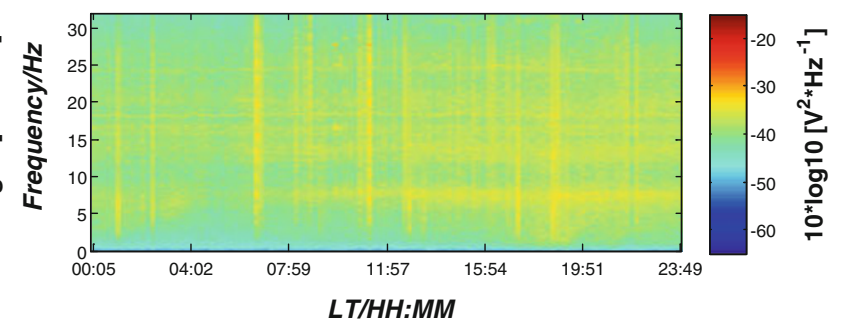

Fig. 1 Power spectra recorded at three stations in China to compare data qualities from different observatories: a, b spectra of $B_{\mathrm{NS}}$ and $B_{\mathrm{EW}}$ components from QJ station; c, d spectra from TH station; and e, f results from YS station

variation characteristics in $B_{\mathrm{NS}}$ and $B_{\mathrm{EW}}$ components, such as in winter (Fig. 2d) when the diurnal shapes are nearly in opposite phases in these two components. The amplitude of $\mathrm{SR}$ in $B_{\mathrm{NS}}$ component is stronger than that in $B_{\mathrm{EW}}$ component in all seasons, which may be caused by an approximate East-West direction distribution of three lightning centers relative to this observatory. In $B_{\mathrm{EW}}$ component, the SR amplitude shows the maximum values in four diurnal curves in four seasons around $16 \mathrm{LT}$ (Beijing Standard Time), corresponding to $08 \mathrm{UT}$. While the first SR mode amplitude in $B_{\mathrm{NS}}$ component shows two or three peaks in a day, such as at 03-05 LT, 16-18 LT, and 21-23 LT, respectively, as shown in Fig. 2a, which corresponds to 19-21 UT, 08-10 UT, and 13-15 UT. Because Asian, African, and American thunderstorm centers play their dominant roles, respectively, in the intervals of 08:00 UT $\pm 1 \mathrm{~h}, 15: 00 \mathrm{UT} \pm 1 \mathrm{~h}$, and 21:00 UT $\pm 1 \mathrm{~h}$ (Hayakawa et al. 2008; Nickolaenko and Hayakawa 2002). Three intervals of intense amplitude in $B_{\mathrm{NS}}$ component were well related to active intervals of three thunderstorm centers. On the other hand, the peak local time with the increasing amplitude in $B_{\mathrm{EW}}$ component was just in accordance with Asian thunderstorm center. The change in diurnal variations of SR demonstrates that the lightning centers play an important role in the observations in four seasons.

\subsubsection{Seasonal variation of SR amplitude}

Another background feature is seasonal variation in SR. Figure 3 presents power spectra in $B_{\mathrm{NS}}$ and $B_{\mathrm{EW}}$ components in a whole year of 2011. The abscissa is the date, and the ordinate is frequency. The color represents SR power spectral density (PSD). The SR-PSD values both in $B_{\mathrm{NS}}$ and $B_{\mathrm{EW}}$ components changed acutely from May to September, and more stably from October to April. This may be related to the seasonal variation of lightning activities mainly from three global lightning centers during May to September. Besides, there are also many impulses occurring during May to September, which may be correlated to those local lightning events during rainy days.

\subsection{Anomalous effect possibly related to 2011 Japan earthquake}

On 11 March 2011, there took place a significant earthquake in Japan with magnitude of 9.0. The earthquake and 
(a) Amplitude of First SR Mode Around March 21st, 2011

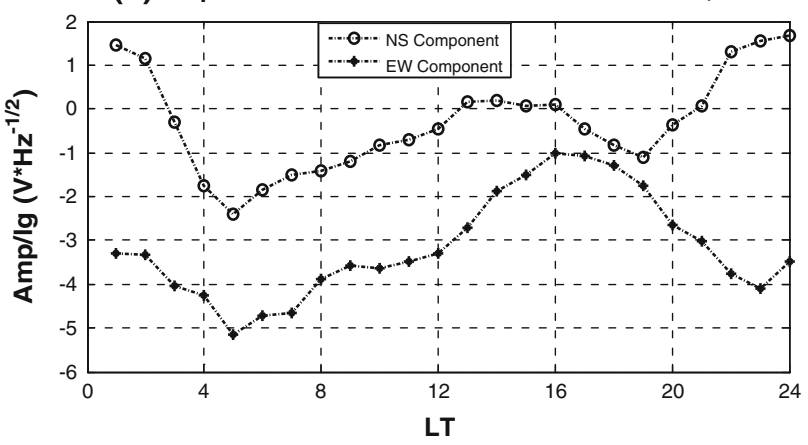

(C) Amplitude of First SR Mode Around September 23rd, 2011

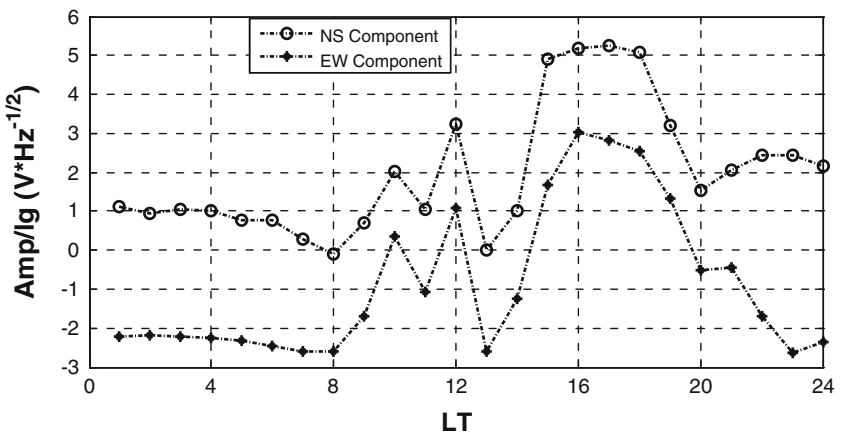

(b) Amplitude of First SR Mode Around June 22nd, 2011

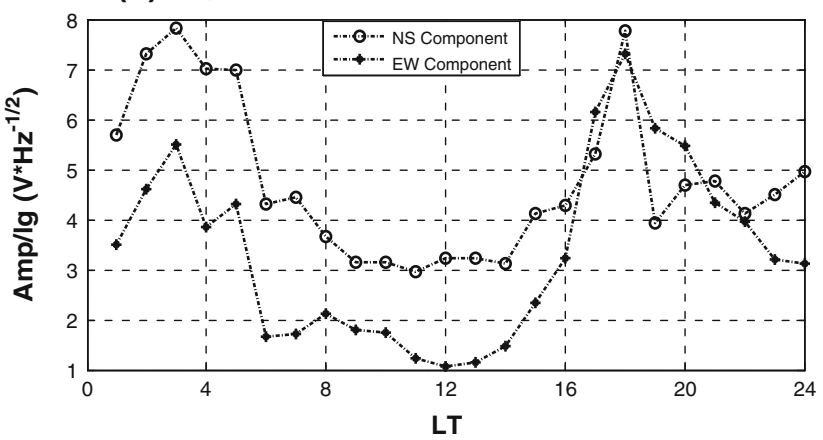

(d) Amplitude of First SR Mode Around December 22nd, 2011

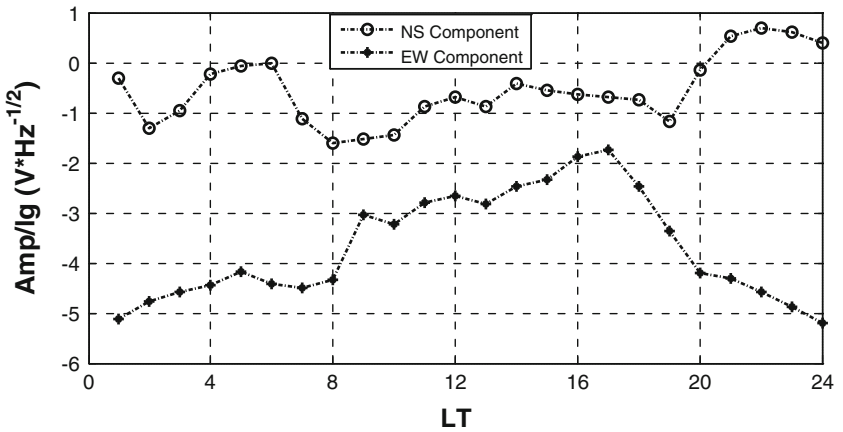

Fig. 2 Amplitude of the first SR mode both in $B_{\mathrm{NS}}$ and $B_{\mathrm{EW}}$ components around equinoxes and solstices. Dashed lines with circles represent the results of $B_{\mathrm{NS}}$ component, and dashed lines with asterisk represent the amplitude of $B_{\mathrm{EW}}$ component

(a) 2011/01/01-2011/12/31 NS Power spectrum

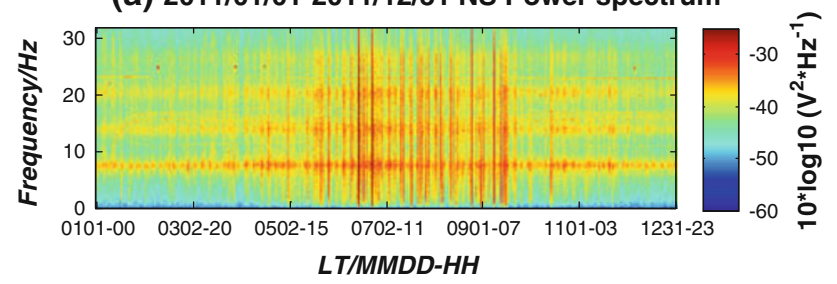

(b) 2011/01/01-2011/12/31 EW Power spectrum

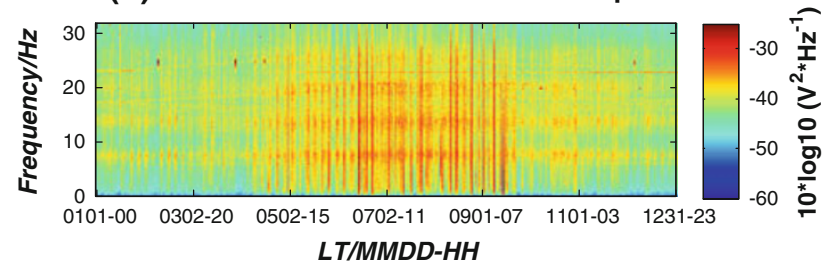

Fig. 3 Power spectra in $B_{\mathrm{NS}}$ and $B_{\mathrm{EW}}$ components in 2011. a, b Represent results of $B_{\mathrm{NS}}$ and $B_{\mathrm{EW}}$ components, respectively

consequent tsunami brought great losses. In this part, We have selected the data 15 days before and 5 days after the earthquake to distinguish anomalous effects possibly related to $M_{\mathrm{S}} 9.0$ Earthquake (epicenter: $38.297^{\circ} \mathrm{N}, 142.372^{\circ} \mathrm{E}$ ) near the east coast of Honshu, Japan on 11 March 2011. The results show that $\mathrm{SR}$ in $B_{\mathrm{NS}}$ and $B_{\mathrm{EW}}$ components presented different features in the aspect of anomalous effects. $B_{\mathrm{EW}}$ component gave us a clear picture of anomalous SR phenomena, which were characterized by increase in amplitude of the lowest four SR modes. Figure 4 displays daily power spectrum 5 days before and 1 day after the earthquake during March 6-12. The amplitude of the lowest four SR modes began to increase 4 days before the earthquake (March 7, see Fig. 4b). And this continued to the day of the earthquake (March 11, see Fig. 4f). Then it recovered to the usual intensity after the earthquake (March 12, see Fig. 4g). We did not find any similar anomalous effects in $B_{\mathrm{NS}}$ component.

In order to confirm that the above anomalous effect is unique in our observation, we have checked daily spectrum in the same period with Japan earthquake in 2012 and 2013. The daily spectra both in $B_{\mathrm{NS}}$ and $B_{\mathrm{EW}}$ components are more affected by local interferences and lightning activities, and we do not find increased amplitude in these two components with same intervals in 2012 and 2013 of pending Japan earthquake. To some extent, this can prove that the anomalous phenomenon is not an ordinary variation of SR, which may correlates to the Japan earthquake.

\section{Discussion}

We have established four SR observatories in the southwestern China since 2010, and we have presented the SR 
(a) 2011/03/06 EW Power spectrum

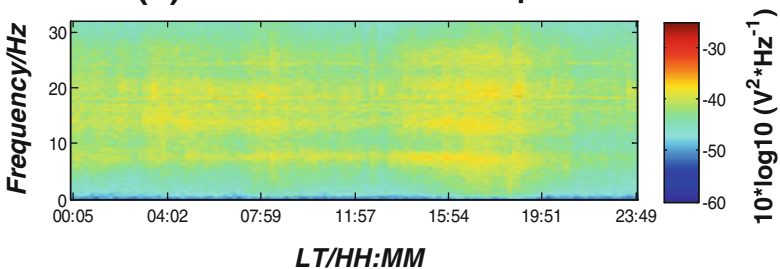

(c) 2011/03/08 EW Power spectrum

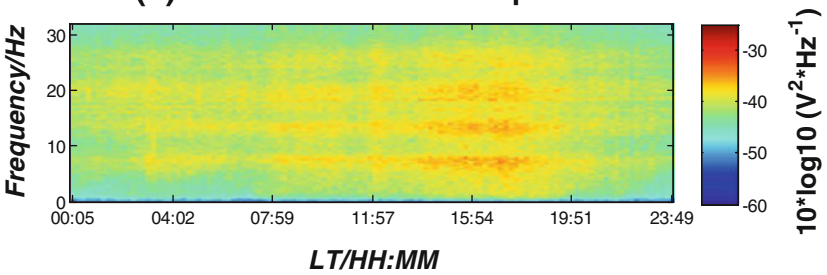

(e) 2011/03/10 EW Power spectrum

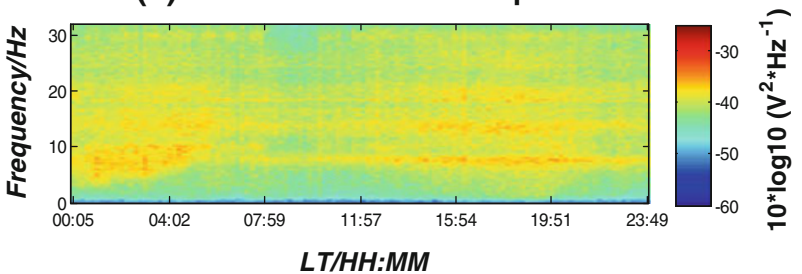

(g) 2011/03/12 EW Power spectrum

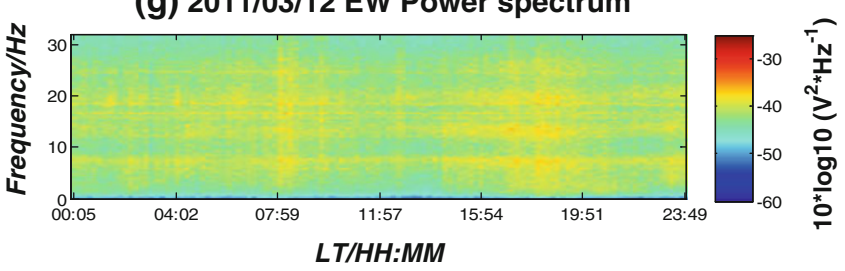

(b) 2011/03/07 EW Power spectrum

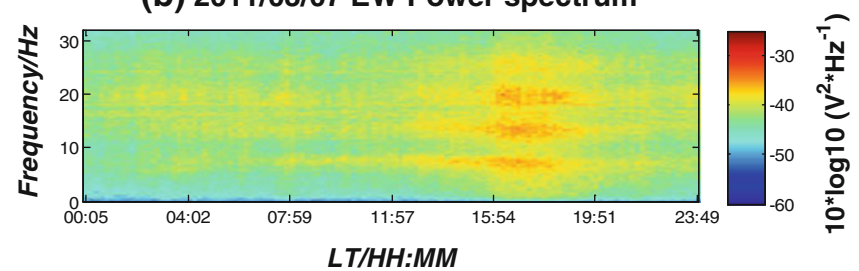

(d) 2011/03/09 EW Power spectrum

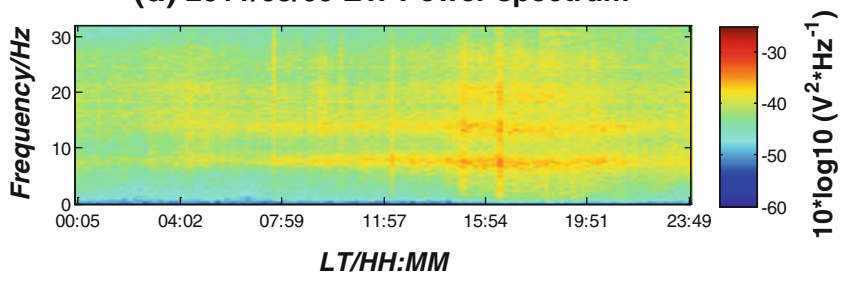

(f) 2011/03/11 EW Power spectrum

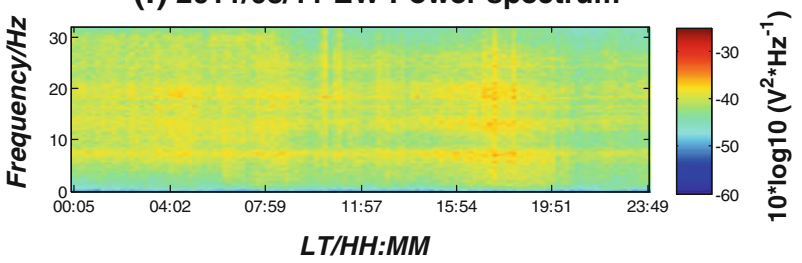

Fig. 4 Daily power spectra in $B_{\mathrm{EW}}$ component 5 days before and 1 day after the 2011 Japan earthquake. SR amplitude increased 4 days before (March 7), and this continued to March 11

data from one observatory of Yongsheng (YS). Some preliminary results on the diurnal and seasonal variations of SR observation at YS are obtained, but further detailed analyses based on a much longer period are highly required in the future. However, it seems that we are successful in finding out a SR anomaly in possible association with the 2011 Japan earthquake. So we will try to discuss the mechanism of this SR anomaly in the following.

\subsection{Geometry of radio propagation}

According to a hypothesis published by Hayakawa et al. (2005), the difference in propagation length between the direct path from any thunderstorm center to the observatory and the disturbed path scattered by the perturbation in the ionosphere over the epicenter is the main cause of wave interference, which manifested in the increased SR amplitude. We tested whether this hypothesis is reasonable even in our case. At first, the position of three thunderstorm centers was defined, referred to an equation published by
Nickolaenko et al. (1998), which gives the varying coordinates of three centers with seasons. We calculated coordinates of three centers in March, and obtained the coordinates of American center at $19^{\circ} \mathrm{S}$ and $70^{\circ} \mathrm{W}$, African center located at $5^{\circ} \mathrm{S}$ and $20^{\circ} \mathrm{E}$, and Asian center at $2.5^{\circ} \mathrm{N}$ and $120^{\circ} \mathrm{E}$, respectively.

We assumed that there was a disturbance in the ionosphere over the epicenter, and we calculated the greatcircle distance of three centers, YS observatory and the disturbance. Figure 5 presents their relative positions. In the case of the Asian source, the direct path from Asian source to the observatory is $3.4 \mathrm{Mm}$, and the disturbed path from the Asian source to the disturbance, then to the observatory is $8.7 \mathrm{Mm}$. The difference of these two paths is 5.3 $\mathrm{Mm}$. The maximum effect of interference is expected when the difference is equal to half of the wavelength. Then we got the wavelength was $10.6 \mathrm{Mm}$. As the wavelength at the first SR mode is $40 \mathrm{Mm}$, the above wavelength corresponded to $40 / 10.6=3.8 \approx 4$ mode number, which was approximately at $26 \mathrm{~Hz}$. 


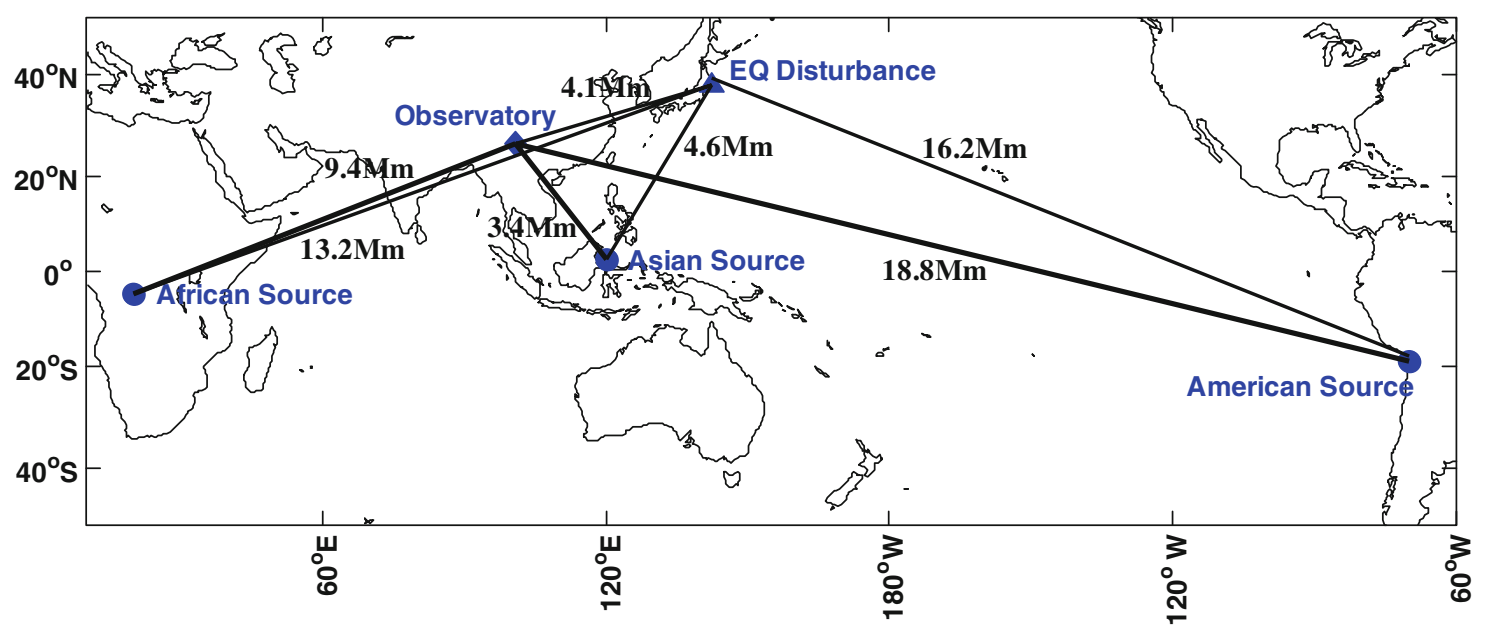

Fig. 5 Relative positions of three thunderstorm centers, YS observatory and earthquake disturbance. The great-circle distance between the direct path and the disturbed path was marked in the figure

Applying the same method to American and African source, we attained the direct path from American source to the observatory was $18.8 \mathrm{Mm}$, and the disturbed path was $20.3 \mathrm{Mm}$. The difference of these two paths was $1.5 \mathrm{Mm}$. The corresponding mode number was 40/ $(2 * 1.5) \approx 13$. Similarly analyzing the African source to obtain the direct path and disturbed path, we got the corresponding SR mode number being 2.5 .

The results illustrate that the mode number obtained from Asian and African center are reasonable, but the interference mode number for the American center is too large and undetectable for our observatory. Moreover, it is noted that the disturbance is positioned behind the observatory along the propagation path of African SourceObservatory, and the effect of such a backscattering is pronounced at ELF observation (Nickolaenko and Hayakawa 2002). Another source, Asian source, is the closest one to the observatory, located nearly in the NS direction relative to the observatory, while the most significant amplitude in $B_{\mathrm{EW}}$ component was detected in the SR experiment at YS station (see Fig. 4). Through above analysis, it can be concluded that African and Asian thunderstorm centers mainly contribute to this anomalous effect by the wave interference between direct paths from these two centers to the observatory and disturbed paths scattered by the ionospheric perturbation over the epicenter.

\subsection{Computational results}

We try to give computational results of SR regular and disturbed spectra in this part. Details on the model can be found in Nickolaenko et al. (2006). We use the regular Mushtak-Williams knee model (regular conductivity profile of the lower ionosphere based on the SR data). The particular knee model was introduced by Mushtak and
Williams (2002), and its application to the SR computations was described by Williams et al. (2006). Seismogenic modifications of conductivity profile and relevant modifications in the SR records were addressed by Hayakawa et al. (2005) and Nickolaenko et al. (2006).

The model supposes that the earthquake causes a local decrease of height of the lower ionosphere, and ELF propagation scattered by this modification. The maximum modification is centered over the epicenter, and the disturbance is axially symmetric and varies with the radius as the Gaussian curve of a given scale diameter $D(D=2,000 \mathrm{~km})$. The distinction of the disturbed profile from the regular one is that the 'knee height' is $55.0 \mathrm{~km}$ in the regular model and $35.0 \mathrm{~km}$ in the disturbed one. Except the $20 \mathrm{~km}$ height depression, all other parameters of the profile remain unchanged.

Figure 6 depicts the computation results of three frames corresponding to three point sources of global thunderstorm activity, i.e., $2.5^{\circ} \mathrm{N}$ and $120^{\circ} \mathrm{E}$ (Asia), $5^{\circ} \mathrm{S}$ and $20^{\circ} \mathrm{E}$ (Africa), $19^{\circ} \mathrm{S}$ and $70^{\circ} \mathrm{W}$ (America). Each frame shows two power spectra of Ez (vertical electric field) component in the frequency of SR band. The black lines represent the power spectra in the regular Earth-ionosphere cavity. The dash-dotted curves show the modified one due to localized ionosphere disturbance.

The upper frame in Fig. 6 corresponds to the Asian thunderstorm center. The disturbed spectrum intensified at three lowest SR modes. Amplitude in SR spectra may increase about $20 \%$. The middle frame in Fig. 6 displays spectra for African thunderstorm center, which shows that the disturbed spectrum increase about $30 \%$ at the second $\mathrm{SR}$ mode relative to the regular one. The third frame shows that the disturbed spectrum for American center increases at the second, third and fourth modes. Because three centers contribute to different SR modes, the computational 

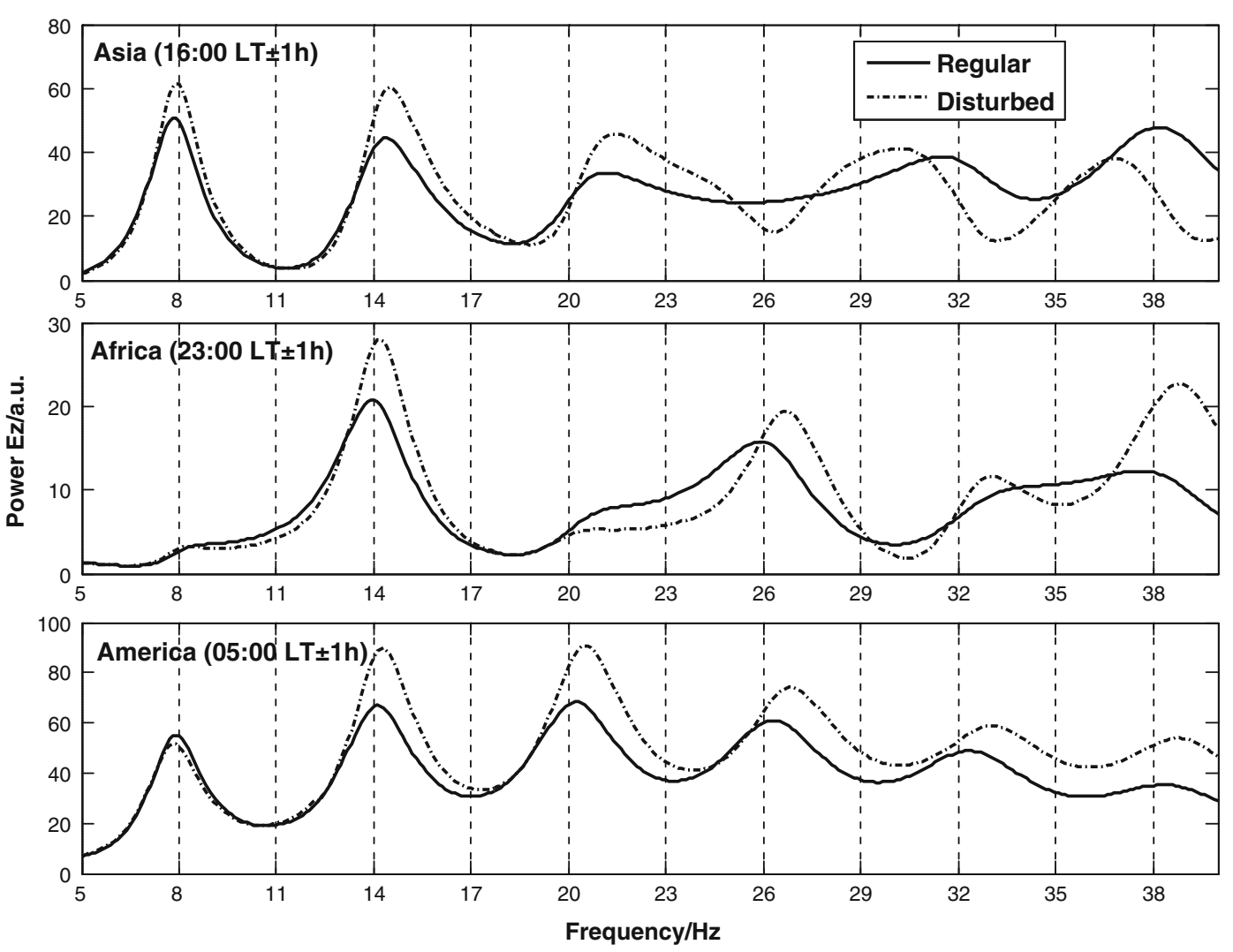

Fig. 6 Regular and disturbed SR spectra in the model of point sources at Asia, Africa, and America

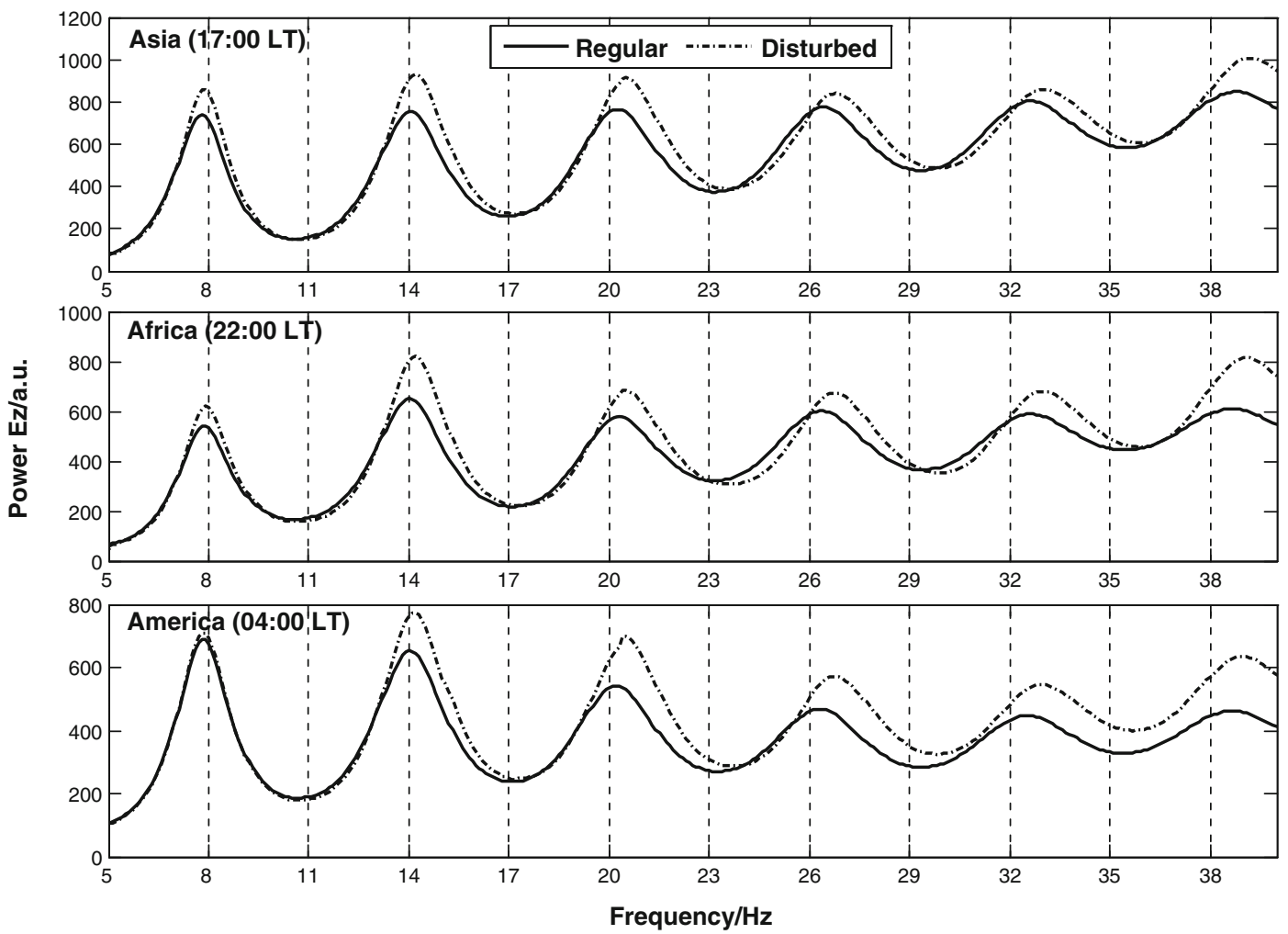

Fig. 7 Regular and disturbed SR spectra for particular intervals of dominant activity at Asia, Africa, and America 
results do not present a persuasive argument which thunderstorm center is dominant.

Twenty-four maps of global distribution of lighting activity were constructed for particular hours (UT) by using the data of Optical Transient Detector (OTD) (Christian et al. 2003; Nickolaenko et al. 2006), and used three leading hour maps corresponding to Asian, African and American thunderstorm centers as a more realistic source models to obtain the SR regular and disturbed spectra. Figure 7 showed this result, and the upper frame corresponded to the time when the Asian thunderstorms dominate in the global activity. The middle and bottom frame showed the results for African center and American center, respectively. We noted that the disturbed spectra increased almost at the lowest four SR modes except the first SR mode for American source.

Figure 7, which is based on a more realistic source model, demonstrated that the localized disturbance over Japan earthquake led to the interference effect which modified the intensity of all lowest four SR modes for the Asian and African source. This is consistent with the analysis of radio propagation geometry (see Sect. 4.1).

As for the possible perturbations in the lower ionosphere, Hayakawa et al. (2012) have already found a clear ionospheric perturbation prior to the Japan earthquake. Similar perturbation in the upper ionosphere has been found by Akhoondzadeh, (2012) and Zolotov et al. (2012), who have found anomalies in GPS TEC within the time interval of 1-3 days before the earthquake after excluding the effects from geomagnetic storms and solar activity.

\section{Conclusions}

In the present study, we used the new observing SR data in China, and analyzed the diurnal and seasonal variations of SR amplitude at YS observatory. Our study exemplified that increased amplitude of SR had a clear association with 2011 Japan earthquake. SR is mainly used in lightning research, but its recent application in earthquake science may bring a new and perspective tool in the field of seismoelectromagnetics. The main conclusions of our research are as follows:

(a) SR amplitude in $B_{\mathrm{NS}}$ component is larger than that in $B_{\mathrm{EW}}$ component. SR in $B_{\mathrm{EW}}$ component is most sensitive to signals from Asian center and SR in $B_{\mathrm{NS}}$ component is in good response to three centers. Amplitude of SR both in $B_{\mathrm{EW}}$ and $B_{\mathrm{NS}}$ components presents seasonal variation.

(b) We found that SR amplitude increased at the lowest four modes several days before 2011 Japan earthquake. Through calculating the direct path from three thunderstorm centers to the observatory and disturbed path scattered by perturbation in the ionosphere, it suggested that Asian and African thunderstorm centers may contribute to this anomalous effect. Modeling results by using more realistic sources were more similar to the observations featured by increase in amplitude at the lowest four SR modes than the results by using three point sources.

A new case of anomalous SR effect related to the large earthquake was presented with new observations in China. This complements the earthquake case study database of anomalous SR. With accumulating of our observations, a statistical study on as many as earthquakes has to be performed in the future. We look forward to repeatability of anomalous SR, or the cause of different features of anomalous SR, and improved hypothesis to explain observed results.

Acknowledgments This study is supported by the Basic Research Project of Institute of Earthquake Science, CEA (2013IES0101, 2010IES0202).

\section{References}

Akhoondzadeh M (2012) Anomalous TEC variations associated with the powerful Tohoku earthquake of 11 March 2011. Nat Hazards Earth Syst Sci 12:1453-1462

Christian HJ, Blakeslee RJ, Boccippio DJ, Boeck WL, Buechler DE, Driscoll KT, Goodman SJ, Hall JM, Koshak WJ, Mach DM, Stewart MF (2003) Global frequency and distribution of lightning as observed from space by the Optical Transient Detector. J Geophys Res 108(D1):4005

Hayakawa M, Ohta K, Nickolaenko AP, Ando Y (2005) Anomalous effect in Schumann resonance phenomena observed in Japan, possibly associated with the Chi-chi earthquake in Taiwan. Ann Geophys 23(4):1335-1346

Hayakawa M, Nickolaenko AP, Sekiguchi M, Yamashita K, Ida Y, Yano M (2008) Anomalous ELF phenomena in the Schumann resonance band as observed at Moshiri (Japan) in possible association with an earthquake in Taiwan. Nat Hazards Earth Syst Sci 8(6):1309-1316

Hayakawa M, Hobara Y, Yasuda Y, Yamaguchi H, Ohta K, Izutsu J, Nakamura T (2012) Possible precursor to the March 11, 2011, Japan earthquake: ionospheric perturbations as seen by subionospheric very low frequency/low frequency propagation. Ann Geophys 55(1):95-99

Mushtak VC, Williams ER (2002) ELF propagation parameters for uniform models of the earth-ionosphere waveguide. J Atmos Solar Terr Phys 64(18):1989-2001

Nickolaenko AP, Hayakawa M (2002) Resonances in the earthionosphere cavity. Kluwer, Dordrecht

Nickolaenko AP, Sátori G, Zieger B, Rabinowicz LM, Kudintseva IG (1998) Parameters of global thunderstorm activity deduced from the long-term Schumann resonance records. J Atmos Solar Terr Phys 60(3):387-399

Nickolaenko A, Hayakawa M, Sekiguchi M, Ando Y, Ohta K (2006) Model modifications in Schumann resonance intensity caused by a localized ionosphere disturbance over the earthquake epicenter. Ann Geophys 24(2):567-575 
Ohta K, Watanabe N, Hayakawa M (2006) Survey of anomalous Schumann resonance phenomena observed in Japan, in possible association with earthquakes in Taiwan. Phys Chem Earth 31(4-9):397-402

Price C, Melnikov A (2004) Diurnal, seasonal and inter-annual variations in the Schumann resonance parameters. J Atmos Solar Terr Phys 66(13-14):1179-1185

Roldugin VC, Maltsev YP, Vasiljev AN, Schokotov AY, Belyajev GG (2004) Diurnal variations of Schumann resonance frequency in NS and EW magnetic components. J Geophys Res 109(A8): A08304

Rossi C, Palangio P, Rispoli F (2007) Investigations on diurnal and seasonal variations of Schumann resonance intensities in the auroral region. Ann Geophys 50(3):301-311

Sátori G, Zieger B (1996) Spectral characteristics of Schumann resonances observed in Central Europe. J Geophys Res 101(D23):29663-29669

Schlegel K, Füllekrug K (2002) 50 years of Schumann resonance. Phys Unserer Zeit 33(6):256-264
Schumann W (1952) Über die strahlungslosen Eigenschwingungen einer leitenden Kugel die von einer Luftschicht und einer Ionosphärenhülle umgeben ist. Zeitschrift Naturforschung Teil A $7: 149$

Sekiguchi M, hobara Y, hayakawa M (2008) Diurnal and seasonal variations in the Schumann resonance parameters at Moshiri, Japan. J Atmos Electr 28(1):1-10

Sentman DD, Fraser BJ (1991) Simultaneous observations of Schumann resonances in California and Australia: evidence for intensity modulation by the local height of the $\mathrm{D}$ region. J Geophys Res 96(A9):15973-15984

Williams ER, Mushtak VC, Nickolaenko AP (2006) Distinguishing ionospheric models using Schumann resonance spectra. J Geophys Res 111(D16):D16107

Zolotov O, Namgaladze A, Prokhorov B (2012) Total electron content disturbances prior to Great Tohoku March 11, 2011 and October 23, 2011 Turkey Van earthquakes and their physical interpretation. arXiv:1205.6118 (Arxiv preprint) 